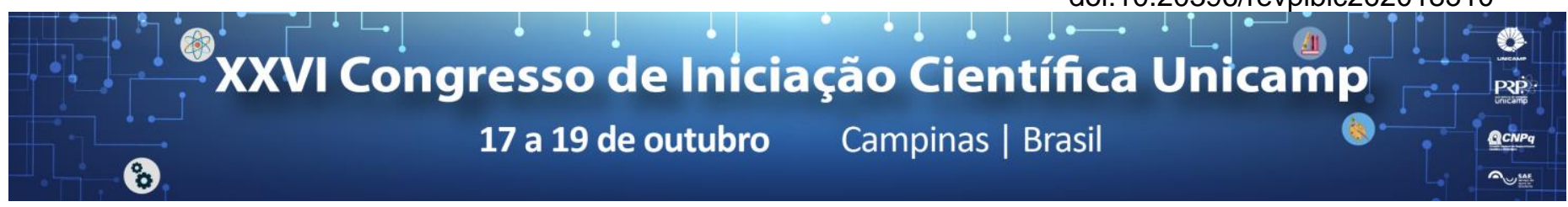

\title{
What is a concept?
}

\section{Ciro Watanabe*, Ruth Elisabeth V. Lopes}

\begin{abstract}
Concepts are the tools we use to understand and interact with the world around us. It is not only useful for communication; concepts are present in most (if not all) cognitive tasks we realize. When we enter a new restaurant, encounter a new animal or meet a new person we automatically start to process information received in the attempt to match them with categories we already have. From an evolutionary point of view, this is really important because if the new object is similar enough with a class we already know, it means that we know how to deal with it (or what to expect from it). However, there is much we still don't know about how concepts represent classes and the different features and functions they display in different domains of knowledge. This is an attempt to investigate the nature of representation and cognition.
\end{abstract}

\section{Key words:}

Concepts, cognition, mind.

\section{Introduction}

Until the 1960s the dominant theory of concepts was the Classical View. It was thought that concepts had a definitional structure, that is, necessary and sufficient conditions of belonging to a category. Clark Hull (1920) defended that a category is individuated by characteristics that are at the same time present in all members (necessary condition) and exclusive of this group, that is, they are not present in other categories (sufficient condition). This view is intuitive because it seems reasonable that members of a class are united by at least one thing they have in common, and that they have something unique that differentiates them from other groups (after all, they are gathered under a general name).

However, after research on family resemblance (see Rosch and Mervis (1975) for a prominent example) an important aspect entered into account in studies of natural language concepts, the typicality. Members of a class are usually located within a gradient of typicality, which is determined by the properties shared by the majority of the group. Thus sparrows are very typical exemplars of the category bird (since the properties they instantiate are shared by most members of their class), while chickens are not (because they have unusual features like being too big etc.). Plus, natural language categories are less like circles (whose edges are well defined) and more like clouds (whose borders are blurry) - there are cases where we arbitrarily decide if an item belongs to a class or not, the borderline cases.

The Classical View didn't predict the typicality effect nor the borderline cases. Thus two other views took place to explain these phenomena, the Prototype and the Exemplar Views. Prototype View defends that categories are represented by summary representations constituted by weighted features according to the frequency they appear in experience (how typical they are). These unified representations are abstracted from any particular exemplar, forming a general schemata (Rumelhart and Ortony 1977). In the Exemplar View, it is assumed that categories are represented by memories of instances that have been previously encountered. Thus my concept bird is the set of birds I have been in touch with.

This research aims to evaluate the different proposals about the nature of our concepts and their adequacy to empirical experiments as well as theoretical competence.

\section{Results and Discussion}

The Exemplar View defends that we categorize new objects by comparing them with previous exemplars we've seen and calculate the degree of similarity between them. The most successful computational model that formalizes this operation is the Generalized Context Model (GCM; Nosofsky, 1984). With GCM we can calculate the psychological distance between a new object $i$ and a known exemplar $j$, which is inversely proportional to their similarity. Then it is possible to extend this to the entire class $J$ of which $j$ belongs. This allows us to estimate the probability of $i$ being categorized as a member of $J$. The Prototype View may easily adapt this model to their theory, by calculating the distance between the new object $i$ and a prototype $k$. Some studies (Voorspoels, 2008) have been made comparing the two models, but nothing conclusive was accomplished.

\section{Conclusions}

Neither Theory is decidedly dismissed, so there is work to be done and experiments to be realized. Also, it is possible that both theories (together with other areas of cognitive science) would be necessary to give a full account of human conceptual structure.

\section{Acknowledgement}

I am grateful to professor Ruth Lopes for the guidance through the last year, and to colleagues from my university, Unicamp.

HULL, C. Quantitative aspects of the evolution of concepts. Psychological Monographs, 1920.

ROSCH, E; MERVIS, C. Family Resemblances: Studies in the Internal Structure of Categories. Cognitive Psychology, California, vol. 7, 573-605, 1975.

NOSOFSKY, R. M. Choice, similarity, and the context theory of classification. Journal of Experimental Psychology:Learning, Memory, and Cognition, 10, 104 114,1984 